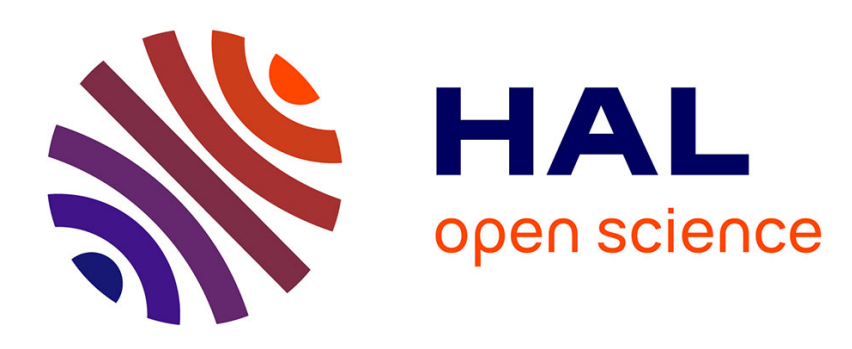

\title{
Effect of turbulent eddy viscosity on the unstable surface mode above an acoustic liner
}

\author{
David Marx, Yves Aurégan
}

\section{To cite this version:}

David Marx, Yves Aurégan. Effect of turbulent eddy viscosity on the unstable surface mode above an acoustic liner. Journal of Sound and Vibration, 2013, 332 (15), pp.3803-3820. 10.1016/j.jsv.2013.02.005 . hal-02540161

\section{HAL Id: hal-02540161 \\ https://hal.science/hal-02540161}

Submitted on 5 Oct 2021

HAL is a multi-disciplinary open access archive for the deposit and dissemination of scientific research documents, whether they are published or not. The documents may come from teaching and research institutions in France or abroad, or from public or private research centers.
L'archive ouverte pluridisciplinaire HAL, est destinée au dépôt et à la diffusion de documents scientifiques de niveau recherche, publiés ou non, émanant des établissements d'enseignement et de recherche français ou étrangers, des laboratoires publics ou privés. 


\title{
Effect of turbulent eddy viscosity on the unstable surface mode above an acoustic liner
}

\author{
David Marx ${ }^{\mathrm{a}, *}$, Yves Aurégan ${ }^{\mathrm{b}}$ \\ ${ }^{a}$ Institut PPRIME - CNRS - Université de Poitiers - ENSMA, Département Fluide \\ Thermique Combustion, ENSIP, Bât. B17, 6 rue Marcel Doré, 86000 Poitiers, France \\ ${ }^{b}$ Laboratoire d'Acoustique de l'Université du Maine (LAUM), Avenue Olivier Messiaen, \\ 72085 Le Mans Cedex 9, France
}

\begin{abstract}
Lined ducts are used to reduce noise radiation from ducts in turbofan engines. In certain conditions they may sustain hydrodynamic instabilities. A local linear stability analysis of the flow in a 2D lined channel is performed using a numerical integration of the governing equations. Several model equations are used, one of them taking into account turbulent eddy viscosity, and a realistic turbulent mean flow profile is used that vanishes at the wall. The stability analysis results are compared to published experimental results. Both the model and the experiments show the existence of an unstable mode, and the importance of taking into account eddy viscosity in the model is shown. When this is done, quantities such as the growth rate and the velocity eigenfunctions are shown to agree correctly.
\end{abstract}

Keywords: duct acoustics, acoustic liner, instability

PACS: 43.28.Py, 43.20.Mv, 43.28.Ra

\footnotetext{
*Corresponding author

Email addresses: david.marx@univ-poitiers.fr (David Marx), yves.auregan@univ-lemans.fr (Yves Aurégan)
} 


\section{Introduction}

Lined ducts are widely used to reduce noise radiation from ducts, for example in air conditioning systems and turbofan engines. In the presence of flow, the interaction of sound and flow above the liner can be complex. One of the main feature of this interaction are nonlinear effects, which have an effect on peak absorption frequency and on sound transmission when the Mach number increases[1, 2]. Another possible effect is the presence of an instability over the liner, which is the subject of the present paper.

In some circumstances, sound amplification by a liner has been observed $[3,4,5]$. This has been the case mostly for plane waves, for low-resistance liners, and for a high enough mean flow velocity. This amplification has been ascribed to an underlying flow instability. In recent experiments [6] the flow has been measured over a liner in such a situation when the liner amplifies sound at some frequency close to the resonance frequency of the liner. The presence of a spatial instability has been confirmed, and quantites such as growth rate, spatial wavelength, and eigenfunctions have been provided. Some numerical evidence of instability over a liner also exist $[7,8]$.

Many analytical works have been performed on propagation modes in lined ducts with flow $[9,10,11,12]$. They often rely on a constant mean flow (or plug flow) assumption. As a result, there is a sheet of vorticity at the wall, and it becomes necessary to introduce a model for enforcing the liner impedance at the wall. A widely used model is that by Myers, which enforces the continuity of particle displacement across the vorticity sheet[13, 14]. Us- 
ing this model, Tester [9] showed that the response of a lined duct to an impulse contains some modes that are amplified during their propagation. This instability was found to be of the convective type. In his classification of modes in lined duct with flow, Rienstra[11], besides acoustic modes, also predicts some unstable hydrodynamic surface modes. Though, the effect of the Myers boundary condition has been questioned $[9,15,16]$. In particular, Brambley[16] has shown that the Myers' boundary condition is ill-posed. The use of classical stability criteria such as Briggs-Bers' $[17,18]$ then becomes invalid. Such a criterion was used by Rienstra[11] to prove the existence of spatially amplified waves. This renders the conclusions about the stability of flow in lined flow ducts fragile. Fortunately, it is possible to avoid the use of the Myers' boundary condition: one has to use a velocity profile that accounts for the boundary layer and vanishes to zero at the wall. It is then not possible to tackle the problem analytically and one has to rely on a numerical solver to calculate the eigenmodes[19, 20, 21, 15, 22, 23], which will be the case here.

The effect of dissipative mechanisms on acoustic propagation and the resulting acoustic damping has been the object of some researches. This effect is well known in the absence of flow, where the wavenumber becomes complex with an imaginary part given by Kirchoff's formula[24]. The presence of flow complicates the matter somewhat[25, 26], and there are still different models for the wavenumbers with flow when dissipation is present [24, 27, 28]. Few works have focused on the effect of viscosity over the modes above an acoustic liner, even is some studies have considered the effect of viscosity on the nature of the boundary condition at a liner[29]. The effect of turbulent 
eddy visosity on the other hand has been taken into account in several studies dealing with the mechanics of an organized wave in a turbulent shear flow $[30,31,32]$, and the same kind of formalism will be used here.

In the present paper, a local 2D linear stability analysis is performed to investigate the presence of an unstable mode over a lined wall in a duct with flow, and the effect of viscous dissipation on the mode is taken into account, which is new to the author knowledge. The different parameters are taken to be identical to those in reference [6], so that the models can be tested against experiments. A brief summary of the experimental results are recalled in section 2. The modelling of the turbulent flow is provided. Several models for the stability analysis are then presented in section 3. These are an inviscid compressible model (Pridmore-Brown), an inviscid incompressible model (Rayleigh), and a viscous incompressible model which takes into account both the molecular and the turbulent eddy viscosity. The numerical solution method is also presented. Section 4 presents the results for the inviscid compressible and incompressible models. These models predict absolute instability and do not match the experiments. Section 5 presents the results for the incompressible but viscous model. This model predicts spatial instability and provides a correct match with the experiments. Finally conclusions are given.

\section{Summary of experimental results}

In Marx et al[33, 6] an instability has been measured in a lined flow duct. The setup and main results are now briefly recalled, and the modelling of 
the mean turbulent flow is presented.

A 2D cut of the experimental configuration is shown Fig. 1. The channel has a height $2 \tilde{H}=0.02 \mathrm{~m}$, with a rigid top wall and a partly-lined bottom wall. In following the tilde $\sim$ is used to indicate physical quantities, while no tilde is used for normalized quantities. Quantities used for normalizing are introduced in the next section. The duct width $(0.08 \mathrm{~m})$ gives an aspect ratio of 4 which, while not very large, we assume is large enough for a 2D model to be suitable. The liner on the bottom wall covers the whole span of the section

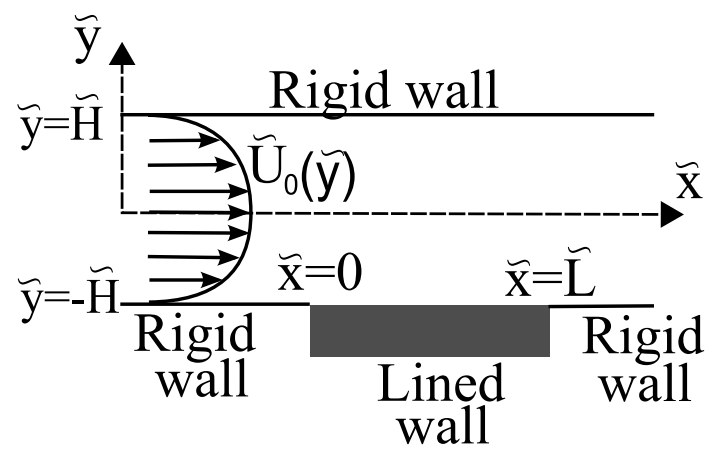

Figure 1: Studied configuration. The notation ${ }^{\sim}$ is for physical quantities

and has a length $\tilde{L}=0.075 \mathrm{~m}$ in the streamwise direction. It consists of tightly spaced square resonators and has the normalized normalized admittance:

$$
Y=-\mathrm{i} Q \tan \left[\tilde{A} \tilde{\omega}+\mathrm{i} \frac{\epsilon}{2}\right]=-\mathrm{i} Q \tan \left[A \omega+\mathrm{i} \frac{\epsilon}{2}\right]
$$

with $\epsilon=0.3, Q=0.8, \tilde{A}=2.5 \times 10^{-4} \mathrm{~s}, A=\tilde{A} \tilde{c}_{0} / \tilde{H}=8.5$. For the stability analysis performed below, the expression for the impedance needs to be continued in the complex $\omega$-plane, and this continuation is valid for the present model[34]. 
The flow velocity $\tilde{U}(\tilde{y})$ is along the $\tilde{x}$-direction and depends on $\tilde{y}$. The velocity profile has been measured at several stations above the liner and its shape changes with streamwise location, with a displacement thickness changing from about $0.9 \mathrm{~mm}$ to $1.5 \mathrm{~mm}$. While the profile just upstream of the liner (above the rigid wall) does not represent the flow at all streamwise stations above the liner, it will be used for the stability analysis. This profile is given in Fig. 2. The section-averaged mean Mach number is defined as:



Figure 2: Experimentally measured mean velocity profile; and analytical approximation obtained by integration of Eq. (3) using Cess' expression for the total viscosity, Eq. (4).

$$
M_{0, \mathrm{avg}}=\frac{1}{2} \int_{-1}^{1} M_{0}(y) \mathrm{d} y
$$

where $M_{0}(y)$ is the Mach number. Its value is: $M_{0, \text { avg }}=0.27$.

When performing the stability analysis, it is desirable to have an analytic form of the velocity profile. In Marx and Aurégan [22], the profile was fitted by using polynomials: a quadratic polynomials in the near wall region, smoothly connected to a quadratic one in the central region. Such a fit ignores the physics of the flow in the near wall region. This region is well 
known to exhibit a viscous sublayer, a buffer layer, and a logarithmic layer. Ignoring these regions seems irrelevant when using a turbulent eddy viscosity model as will be done below. Thus a profile physically acceptable in the near wall region will be used in the following even if it is not as good as the fit in [22] for the central region of the channel. Note that in the literature the flow in the wall region has been measured mainly for flow over rigid or possibly rough surfaces, but not for the kind of wall corresponding to the acoustic liners. This is another reason for using the velocity profile just upstream of the acoustic material, since it is is measured over a rigid wall.

The mean flow used in the present work is obtained by integrating the following expression $[30,32]$ :

$$
\frac{\mathrm{d} U_{0}^{+}}{\mathrm{d} y}=-\frac{\operatorname{Re}_{\tau} y}{\nu_{T}^{+}(y)}
$$

The + superscript indicates a normalization in wall units. In such a normalization the velocity is normalized by the wall friction velocity, $\tilde{u}_{\tau}$; the total viscosity, $\tilde{\nu}_{T}(y)$, is normalized by the physical viscosity, $\tilde{\nu}$; and the Reynolds number is given by $\operatorname{Re}_{\tau}=\tilde{u}_{\tau} \tilde{H} / \tilde{\nu}$. The total viscosity is the sum of the physical viscosity and of the turbulent eddy viscosity, $\tilde{\nu}_{t}$, that is:

$$
\tilde{\nu}_{T}=\tilde{\nu}+\tilde{\nu}_{t} \quad \text { or } \quad \nu_{T}^{+}=1+\nu_{t}^{+}
$$

It is given by Cess' expression [30, 31, 32]:

$$
\nu_{T}^{+}(y)=\frac{1}{2}+\frac{1}{2}\left[1+\frac{\kappa^{2} \operatorname{Re}_{\tau}^{2}}{9}\left(1-y^{2}\right)^{2}\left(1+2 y^{2}\right)^{2}\left(1-\mathrm{e}^{(|y|-1) \operatorname{Re}_{\tau} / A_{V D}}\right)^{2}\right]
$$

$\kappa=0.42$ is the von Karman constant, and $A_{V D}=25.4$ is a constant. This expression is valid for $\operatorname{Re}_{\tau}$ up to a few thousand, which is the case here. This 
model is based on a turbulent eddy viscosity using a Prandtl mixing layer hypothesis and a Van Driest correction. It includes the viscous sublayer, the buffer layer, the logarithmic layer and a wake correction at the center of the channel. These features have also been used by Agarwal and Bull [20] in their study of acoustic modes in a cylindrical duct, while with a slightly different model. These authors did not include the effect of viscosity on the perturbation though. The previous expressions allow for a degree of freedom, which is $\operatorname{Re}_{\tau}$, or likewise, $\tilde{u}_{\tau}$. These are determined so that the experimental profile and the analytical profile correspond to the same sectionaveraged Mach number of 0.27 , which provides: $\operatorname{Re}_{\tau}=2760$, corresponding to $\tilde{u}_{\tau}=4.14 \mathrm{~m} \cdot \mathrm{s}^{-1}$. The analytical velocity profile obtained by integrating Eq. (3) together with Eq. (4) using this value for $\operatorname{Re}_{\tau}$ is given in Fig. 2. It matches correctly the experimentally measured profile. The total eddy viscosity is given in Fig. 3. It is seen that the turbulence is generating viscosity more than 200 times that of the molecular viscosity.

For a high enough flow speed, the acoustic transmission by the liner of acoustic waves in a frequency range around the liner resonance frequency was abnormally large [6]. Some phase-locked laser measurements of the flow were performed and structures convected by the flow and growing spatially were observed above the liner. An example of fluctuating axial velocity, $\tilde{u}^{\prime}$, and fluctuating transverse velocity, $\tilde{v}^{\prime}$, at one phase is shown in Fig. 4. This was obtained for a section-averaged mean Mach number $M_{0, \text { avg }}=0.27$ and an incident acoustic wave with frequency $\tilde{f}_{a}=1060 \mathrm{~Hz}$ at sound pressure level $L_{i}=133 \mathrm{~dB}$. The normalized fluctuating velocity could be put into the form 


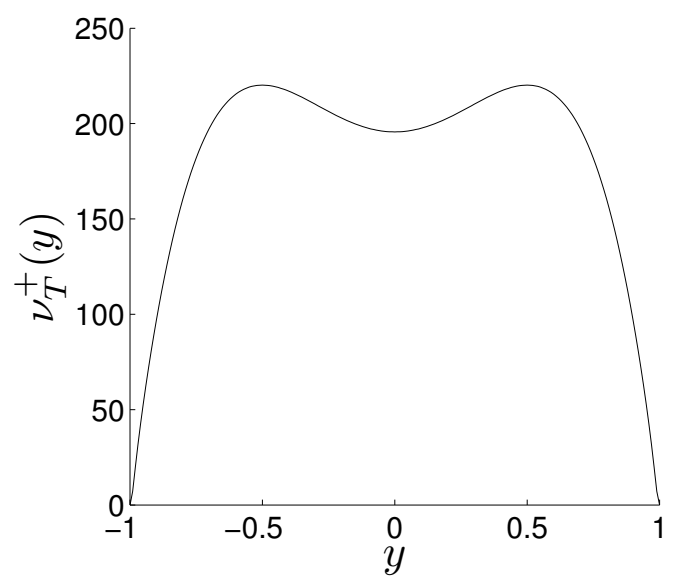

Figure 3: Total viscosity, given by Eq. (4) for $\operatorname{Re}_{\tau}=2760$.
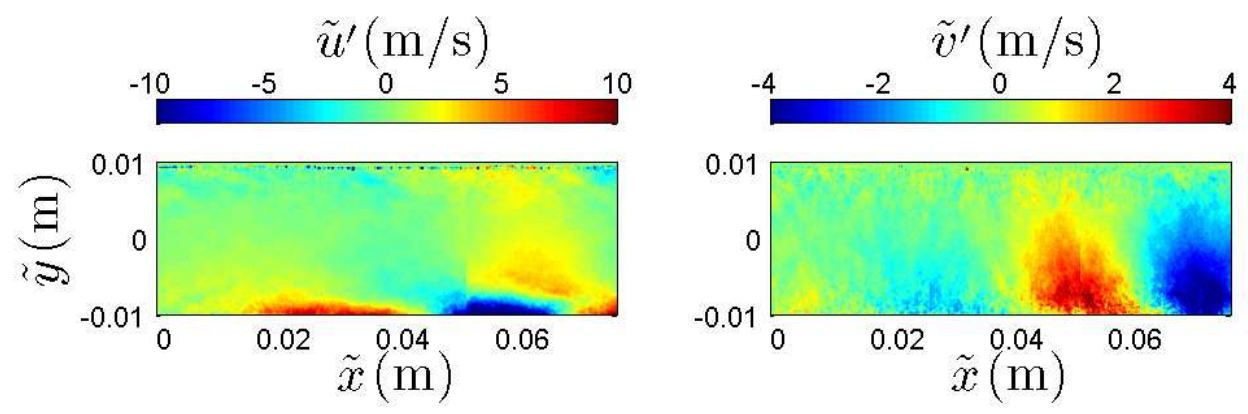

Figure 4: Maps of the phase-averaged axial $\left(\tilde{u}^{\prime}\right)$ and transverse $\left(\tilde{v}^{\prime}\right)$ velocity over the height of the channel. The liner covers the bottom wall, and the origin $\tilde{x}=0$ is counted from the position where the liner starts.

of a travelling wave amplifying in the downstream direction. That is:

$$
u^{\prime}(x, y, t)=\hat{u}\left(y, \alpha, \omega_{a}\right) \mathrm{e}^{\mathrm{i}\left(\alpha x-\omega_{a} t\right)} \quad v^{\prime}(x, y, t)=\hat{v}\left(y, \alpha, \omega_{a}\right) \mathrm{e}^{\mathrm{i}\left(\alpha x-\omega_{a} t\right)}
$$

Here $\alpha=\alpha_{r}+\mathrm{i} \alpha_{i}$ is the complex wavenumber $\left(-\alpha_{i}\right.$ is the growth rate). The normalized angular frequency $\omega_{a}$ corresponds to the particular frequency of the exciting incident acoustic wave $\left(\omega_{a} \sim 0.196\right)$. The eigenfunctions $\hat{u}$ and 
$\hat{v}$ can also be written:

$$
\hat{u}(y)=|\hat{u}(y)| \mathrm{e}^{\mathrm{i} \phi_{u}(y)} \quad \hat{v}(y)=|\hat{v}(y)| \mathrm{e}^{\mathrm{i} \phi_{v}(y)}
$$

In Marx et al[33, 6], the wave number has been given to be $\alpha=1.3-0.3 \mathrm{i}$ (corresponding to $\tilde{\alpha}_{r} \sim 130 \mathrm{~m}^{-1}$ and $\tilde{\alpha}_{i} \sim 30 \mathrm{~m}^{-1}$ ), and the eigenfunctions $|\hat{u}(y)|$ and $|\hat{v}(y)|$ have also been given. These will be compared to the result of the stability analysis in the following.

\section{Model and stability analysis}

\subsection{Compressible inviscid model}

A usual model for investigating the modes in duct acoustics are the compressible Euler, the mass conservation, and the entropy conservation equations, assuming isentropic movement $[9,10,11,12]$ :

$$
\frac{\partial \tilde{\rho}}{\partial \tilde{t}}+\tilde{\nabla} \cdot(\tilde{\rho} \tilde{\mathbf{u}})=0 \quad \tilde{\rho} \frac{\partial \tilde{\mathbf{u}}}{\partial \tilde{t}}+\tilde{\rho} \tilde{\mathbf{u}} \cdot \tilde{\nabla} \tilde{\mathbf{u}}=-\tilde{\nabla} \tilde{p} \quad \frac{\mathrm{d} \tilde{p}}{\mathrm{~d} \tilde{t}}=\tilde{c}_{0}^{2} \frac{\mathrm{d} \tilde{\rho}}{\mathrm{d} \tilde{t}}
$$

where $\tilde{\mathbf{u}}=(\tilde{u}, \tilde{v})$ is the velocity vector, $\tilde{\rho}$ is the density, $\tilde{p}$ is the pressure. According to the previous section, a 2D model is considered. In order to perform a linear stability analysis, these equations are linearized. For this we introduce the following splitting of the variables: $\tilde{\mathbf{u}}(\tilde{x}, \tilde{y}, \tilde{t})=\tilde{\mathbf{U}}_{\mathbf{0}}(\tilde{y})+$ $\tilde{\mathbf{u}}^{\prime}(\tilde{x}, \tilde{y}, \tilde{t}) ; \tilde{p}(\tilde{x}, \tilde{y}, \tilde{t})=\tilde{p}_{0}+\tilde{p}^{\prime}(\tilde{x}, \tilde{y}, \tilde{t}) ; \tilde{\rho}(\tilde{x}, \tilde{y}, \tilde{t})=\tilde{\rho}_{0}+\tilde{\rho}^{\prime}(\tilde{x}, \tilde{y}, \tilde{t})$. The subscript 0 refers to the base flow and the prime represents a fluctuation upon this base flow. The mean base flow $\tilde{\mathbf{U}}_{\mathbf{0}}=\left(\tilde{U}_{0}(\tilde{y}), 0\right)$ is in the $\tilde{x}$-direction. It is allowed to depend on $\tilde{y}$ so that a mean shear is possibly taken into account. The set 
of linearized equations for the perturbations $\tilde{u}^{\prime}, \tilde{v}^{\prime}, \tilde{p}^{\prime}$ can be reduced to:

$$
\begin{array}{r}
\frac{\partial \tilde{u}^{\prime}}{\partial \tilde{t}}+\tilde{U}_{0} \frac{\partial \tilde{u}^{\prime}}{\partial \tilde{x}}+\tilde{v}^{\prime} \frac{\mathrm{d} \tilde{U}_{0}}{\mathrm{~d} \tilde{y}}=-\frac{1}{\tilde{\rho}_{0}} \frac{\partial \tilde{p}^{\prime}}{\partial \tilde{x}} \\
\frac{\partial \tilde{v}^{\prime}}{\partial \tilde{t}}+\tilde{U}_{0} \frac{\partial \tilde{v}^{\prime}}{\partial \tilde{x}}=-\frac{1}{\tilde{\rho}_{0}} \frac{\partial \tilde{p}^{\prime}}{\partial \tilde{y}} \\
\left(\frac{\partial \tilde{p}^{\prime}}{\partial \tilde{t}}+\tilde{U}_{0} \frac{\partial \tilde{p}^{\prime}}{\partial \tilde{x}}\right)+\tilde{\rho}_{0} \tilde{c}_{0}^{2}\left(\frac{\partial \tilde{u}^{\prime}}{\partial \tilde{x}}+\frac{\partial \tilde{v}^{\prime}}{\partial \tilde{y}}\right)=0
\end{array}
$$

For every variable a modal solution of the form $\tilde{u}^{\prime}(y)=\hat{\tilde{u}}(\tilde{y}, \tilde{\alpha}, \tilde{\omega}) \mathrm{e}^{\mathrm{i}(\tilde{\alpha} \tilde{x}-\tilde{\omega} \tilde{t})}$ is introduced, where the wavenumber, $\tilde{\alpha}=\tilde{\alpha}_{r}+\mathrm{i} \tilde{\alpha}_{i}$, and the angular frequency, $\tilde{\omega}=\tilde{\omega}_{r}+\mathrm{i} \tilde{\omega}_{i}$, are both complex. All variables are then normalized using for the length scale the channel half-width $\tilde{H}$; for the velocity scale the speed of sound $\tilde{c}_{0}$; for the density scale the mean unperturbed density $\tilde{\rho}_{0}$; and for the pressure scale $\tilde{\rho}_{0} \tilde{c}_{0}^{2}$. This gives: $y=\tilde{y} / \tilde{H} ; x=\tilde{x} / \tilde{H} ; \alpha=\tilde{\alpha} \tilde{H} ; \omega=\tilde{\omega} \tilde{H} / \tilde{c}_{0}$ $; \hat{u}=\hat{\tilde{u}} / \tilde{c}_{0} ; \hat{v}=\hat{\tilde{v}} / \tilde{c}_{0} ; \hat{p}=\hat{\tilde{p}} /\left(\tilde{\rho}_{0} \tilde{c}_{0}^{2}\right)$. The normalized modal solution is then $u^{\prime}(y)=\hat{u}(y, \alpha, \omega) \mathrm{e}^{\mathrm{i}(\alpha x-\omega t)}$ and the following system is finally obtained for the modal amplitudes:

$$
\begin{array}{r}
\mathrm{i}\left(\alpha M_{0}-\omega\right) \hat{u}+\hat{v} \frac{\mathrm{d} M_{0}}{\mathrm{~d} y}=-\mathrm{i} \alpha \hat{p} \\
\mathrm{i}\left(\alpha M_{0}-\omega\right) \hat{v}=-\frac{\mathrm{d} \hat{p}}{\mathrm{~d} y} \\
\mathrm{i}\left(\alpha M_{0}-\omega\right) \hat{p}+\left(\mathrm{i} \alpha \hat{u}+\frac{\mathrm{d} \hat{v}}{\mathrm{~d} y}\right)=0
\end{array}
$$

where $M_{0}(y)=\tilde{U}_{0}(y) / \tilde{c}_{0}$ is the Mach number. Note that it is possible to combine these equations to obtain the well known Pridmore-Brown equation for calculating compressible modes uppon a sheared mean flow:

$$
\frac{\mathrm{d}^{2} \hat{p}}{\mathrm{~d} y^{2}}-\left[\frac{2 \alpha}{\left(\alpha M_{0}-\omega\right)} \frac{\mathrm{d} M_{0}}{\mathrm{~d} y}\right] \frac{\mathrm{d} \hat{p}}{\mathrm{~d} y}-\left[\alpha^{2}-\left(\alpha M_{0}-\omega\right)^{2}\right] \hat{p}=0
$$




\subsection{Incompressible inviscid model}

Let us introduce the incompressible equivalent to the compressible model of the previous section. It is obtained by replacing $\frac{\partial \tilde{\rho}}{\partial \tilde{t}}+\tilde{\nabla} \cdot(\tilde{\rho} \tilde{\mathbf{u}})=0$ in Eq. (7) by $\tilde{\nabla} \cdot(\tilde{\rho} \tilde{\mathbf{u}})=0$. This gives for the modal amplitudes:

$$
\begin{array}{r}
\mathrm{i}\left(\alpha M_{0}-\omega\right) \hat{u}+\hat{v} M_{0}^{\prime}=-\mathrm{i} \alpha \hat{p} \\
\mathrm{i}\left(\alpha M_{0}-\omega\right) \hat{v}=-\frac{\mathrm{d} \hat{p}}{\mathrm{~d} y} \\
\mathrm{i} \alpha \hat{u}+\frac{\mathrm{d} \hat{v}}{\mathrm{~d} y}=0
\end{array}
$$

The velocity used for the normalization is still the numerical value of the speed of sound $\tilde{c}_{0}$ of the medium if it were compressible. This is the reason why the normalized velocity still appears as a Mach number, $M_{0}$. At first sight this normalization may appear not very relevant for an incompressible study but this choice will allow a direct comparison with the inviscid compressible case (and of course it is correct in terms of numerical value).

It is also possible to combine these equations to obtain the Rayleigh equation:

$$
\frac{\mathrm{d}^{2} \hat{p}}{\mathrm{~d} y^{2}}-\left[\frac{2 \alpha}{\left(\alpha M_{0}-\omega\right)} \frac{\mathrm{d} M_{0}}{\mathrm{~d} y}\right] \frac{\mathrm{d} \hat{p}}{\mathrm{~d} y}-\alpha^{2} \hat{p}=0
$$

Note that the equation given here involves the pressure variable and is a bit different from the more frequently encountered form involving the streamfunction and a double derivative of the mean velocity with respect to $y$.

\subsection{Incompressible viscous model}

As found in [22] and as shown below, it is important to account for the effect of viscosity on the unstable mode. When dealing with acoustic modes, viscous and thermal losses are both equally important. Taking into 
account losses requires one to use an energy equation. As will be argued below, the unstable mode is hydrodynamic in the sense that it is not much affected by compressibility. This is the reason why an incompressible model will be used for studying the effect of viscosity, and no energy equation is required. As in $[30,31,32]$, the stability analysis concerns some coherent modes superimposed on a turbulence whose effect is to add a kinematic (eddy) viscosity. As seen in Fig. 3, at the high Reynolds number investigated here this eddy viscosity is large compared to the molecular viscosity except in the close vicinity to the wall. Of course, the turbulent eddy viscosity depends on $y$. Hence, the 2D incompressible viscous linearized Navier-Stokes equations with a variable viscosity are introduced. For the physical variables, these are [32]:

$$
\begin{array}{r}
\frac{\partial \tilde{u}^{\prime}}{\partial \tilde{t}}+\tilde{U}_{0} \frac{\partial \tilde{u}^{\prime}}{\partial \tilde{x}}+\tilde{v}^{\prime} \frac{\mathrm{d} \tilde{U}_{0}}{\mathrm{~d} \tilde{y}}=-\frac{1}{\tilde{\rho}_{0}} \frac{\partial \tilde{p}^{\prime}}{\partial \tilde{x}}+\frac{\mathrm{d} \tilde{\nu_{T}}}{\mathrm{~d} \tilde{y}} \frac{\partial \tilde{u}^{\prime}}{\partial \tilde{y}}+\frac{\mathrm{d} \tilde{\nu_{T}}}{\mathrm{~d} \tilde{y}} \frac{\partial \tilde{v}^{\prime}}{\partial \tilde{x}}+\tilde{\nu_{T}}\left(\frac{\partial^{2} \tilde{u}^{\prime}}{\partial \tilde{x}^{2}}+\frac{\partial^{2} \tilde{u}^{\prime}}{\partial \tilde{y}^{2}}\right) \\
\frac{\partial \tilde{v}^{\prime}}{\partial \tilde{t}}+\tilde{U}_{0} \frac{\partial \tilde{v}^{\prime}}{\partial \tilde{x}}=-\frac{1}{\tilde{\rho}_{0}} \frac{\partial \tilde{p}^{\prime}}{\partial \tilde{y}}+2 \frac{\mathrm{d} \tilde{\nu_{T}}}{\mathrm{~d} \tilde{y}} \frac{\partial \tilde{v}^{\prime}}{\partial \tilde{y}}+\tilde{\nu_{T}}\left(\frac{\partial^{2} \tilde{v}^{\prime}}{\partial \tilde{x}^{2}}+\frac{\partial^{2} \tilde{v}^{\prime}}{\partial \tilde{y}^{2}}\right) \\
\frac{\partial \tilde{u}^{\prime}}{\partial \tilde{x}}+\frac{\partial \tilde{v}^{\prime}}{\partial \tilde{y}}=0
\end{array}
$$

where as before $\tilde{\nu_{T}}(\tilde{y})$ is the total viscosity, including molecular and turbulent contributions. By combining these equations, one could obtain the modified Orr-Sommerfeld equation initially proposed by Reynolds and Hussain [31, Eq 6.1] for calculating the modes of an organized wave in a turbulent medium. A model is needed for this viscosity, and the expression by Cess, Eq. (4), will be used. It is recalled that $\tilde{U}_{0}$ is obtained by integrating Eq. (3), so that the mean velocity and the total viscosity model are consistent, which was not the case in $[22]$. 
As before modal solutions are introduced and the previous equations are normalized. As in the previous section (see remark below Eqs. (15-17)) the velocity for the normalization is the numerical value of the speed of sound $\tilde{c}_{0}$ if the medium were compressible. By gathering the powers of $\alpha$ the following set of equations is obtained:

$$
\begin{array}{r}
\left(-\mathrm{i} \omega \hat{u}+\hat{v} M_{0}^{\prime}-\frac{1}{\operatorname{Re}} \nu_{T}^{+} \frac{\mathrm{d}^{2} \hat{u}}{\mathrm{~d} y^{2}}-\frac{1}{\operatorname{Re}} \frac{\mathrm{d} \nu_{T}^{+}}{\mathrm{d} y} \frac{\mathrm{d} \hat{u}}{\mathrm{~d} y}\right) \\
+\alpha\left(\mathrm{i} M_{0} \hat{u}+\mathrm{i} \hat{p}-\frac{1}{\operatorname{Re}} \frac{\mathrm{d} \nu_{T}^{+}}{\mathrm{d} y} \mathrm{i} \hat{v}\right)+\alpha^{2}\left(\frac{1}{\operatorname{Re}} \nu_{T}^{+} \hat{u}\right)=0 \\
\left(-\mathrm{i} \omega \hat{v}+\frac{\mathrm{d} \hat{p}}{\mathrm{~d} y}-\frac{1}{\operatorname{Re}} \nu_{T}^{+} \frac{\mathrm{d}^{2} \hat{v}}{\mathrm{~d} y^{2}}-\frac{2}{\operatorname{Re}} \frac{\mathrm{d} \nu_{T}^{+}}{\mathrm{d} y} \frac{\mathrm{d} \hat{v}}{\mathrm{~d} y}\right)+\alpha\left(\mathrm{i} M_{0} \hat{v}\right)+\alpha^{2}\left(\frac{1}{\operatorname{Re}} \nu_{T}^{+} \hat{v}\right)=0 \\
\frac{\mathrm{d} \hat{v}}{\mathrm{~d} y}+\alpha(\mathrm{i} \hat{u})=0(2)
\end{array}
$$

The Reynolds number is defined by $\operatorname{Re}=\tilde{H} \tilde{c}_{0} / \tilde{\nu}$. The total viscosity is normalized in the same way as in section 2 .

\subsection{Boundary conditions}

To solve the equations governing the flow, boundary conditions are needed. The liner at the bottom wall is characterized by its admittance $Y(\omega)$ (or its impedance $Z(\omega)$ ), which provides a relation between the pressure and the wall normal velocity. Using the same normalization as before, as well as the following one:

$$
Y=\tilde{\rho}_{0} \tilde{c}_{0} \tilde{Y}
$$

we have at the bottom wall at $y=-1$ :

$$
\hat{v}(-1, \alpha, \omega)=-Y(\omega) \hat{p}(-1, \alpha, \omega) \quad \forall \alpha, \omega
$$


This relation can be used when the mean flow velocity satisfies $M_{0}(-1)=0$. If there is a slip velocity at the wall, that is if $M_{0}(-1) \neq 0$ (as is the case when the plug flow assumption, $M_{0}=$ cst, is used), one needs to account for the vorticity sheet at the wall, and the boundary condition becomes the Myers' boundary condition:

$$
\omega \hat{v}(-1, \alpha, \omega)=Y(\omega)\left(\alpha M_{0}(-1)-\omega\right) \hat{p}(-1, \alpha, \omega) \quad \forall \alpha, \omega
$$

When $M_{0}(-1)=0$ relations (25) and (26) are equivalent. Hence, we can use only the latter one. But according to the introduction, when $M_{0}(-1) \neq 0$, the Myers boundary condition is not "well-posed", and usual criteria for stability are not applicable. We will nevertheless use this condition for purposes of validation.

At the upper rigid wall (at $y=1$ ), the admittance is zero, we have:

$$
\hat{v}(1, \alpha, \omega)=0 \quad \forall \alpha, \omega
$$

In the viscous case, a non-slip velocity is enforced at both walls:

$$
\hat{u}( \pm 1, \alpha, \omega)=0 \quad \forall \alpha, \omega
$$

\subsection{Stability problem and solution method}

\subsubsection{Generalized eigenvalue problem}

In the inviscid compressible case, we have to solve the system (11-13) with boundary conditions (26-27). In the viscous incompressible case, we have to solve the system (22-24) with boundary conditions (26-28). In either case, the problem is an eigenvalue problem, which can be solved only for some couples $(\alpha, \omega)$, that is, when $\alpha$ and $\omega$ satisfy the dispersion relation $D(\alpha, \omega)=0$. 
As the problem cannot be solved analytically, a numerical solution method is used. The problem is discretized in the $y$-direction using a spectral Chebyshev collocation method $[35,36]$ and $\mathrm{N}+1$ collocation points. The collocation points are of the Gauss-Lobato type[35, 36]: $y_{j}=\cos (j \pi / N)$ for $j=0 \ldots N$. On the grid thus defined, we define the vectors of unknowns:

$$
\mathbf{U}=\left[\begin{array}{llll}
\hat{u}_{0} & \hat{u}_{1} & \ldots & \hat{u}_{N}
\end{array}\right]^{t} ; \mathbf{V}=\left[\begin{array}{llll}
\hat{v}_{0} & \hat{v}_{1} & \ldots & \hat{v}_{N}
\end{array}\right]^{t} \quad ; \mathbf{P}=\left[\begin{array}{llll}
\hat{p}_{0} & \hat{p}_{1} & \ldots & \hat{p}_{N}
\end{array}\right]^{t}
$$

where []$^{t}$ denotes the transpose. These vectors contain the value of the variables at the collocation points. Hence, for example, $\hat{u}_{0}$ is the value of $\hat{u}$ at collocation point $y_{0}=1$, and $\hat{u}_{N}$ is the value of $\hat{u}$ at collocation point $y_{N}=-1$. The y-derivative of a vector is obtained by multiplying this vector by a suitable differentiation square matrix $\mathbf{D}$ of size $N+1[35,36]$. This procedure yields a generalized eigenvalue problem that is now specified for the different cases under consideration.

In the inviscid compressible case, discretizing the system (11-13), one obtains the following generalized eigenvalue problem:

$$
(\underbrace{\left[\begin{array}{ccc}
-\mathrm{i} \omega \mathbf{I} & \mathbf{M}_{\mathbf{0}}^{\prime} & \\
-\mathbf{i} \omega \mathbf{I} & \mathbf{D} \\
\mathbf{D} & -\mathbf{i} \omega \mathbf{I}
\end{array}\right]}_{\mathbf{A}}+\alpha \underbrace{\left[\begin{array}{lll}
\mathrm{i} \mathbf{M}_{\mathbf{0}} & & \mathrm{iI} \\
& \mathbf{i M}_{\mathbf{0}} & \\
\mathrm{i} & & \mathrm{i} \mathbf{M}_{\mathbf{0}}
\end{array}\right]}_{\mathbf{B}} \cdot\left[\begin{array}{l}
\mathbf{U} \\
\mathbf{V} \\
\mathbf{P}
\end{array}\right]=0
$$

$\mathbf{A}$ and $\mathbf{B}$ are square matrix of size $3 N+3 ; \mathbf{M}_{\mathbf{0}}$ is the diagonal matrix (of size $N+1$ ) with the values of the mean velocity at the collocation points on the diagonal; $\mathbf{M}_{\mathbf{0}}^{\prime}$ is also a diagonal matrix (of size $N+1$ ) containing the values of the derivative of the mean velocity at the collocation points; $\mathbf{I}$ is the identity 
matrix (of size $N+1$ ). Parts left blank are filled with zeros. One has still to take into account the boundary conditions (26-27). This is done by replacing the rows $\mathrm{N}+2$ and $2 N+2$ of the matrices $\mathbf{A}$ and $\mathbf{B}$ by the discretized form of the boundary conditions.

In the inviscid incompressible case, discretizing the system (15-17) gives:

$$
(\underbrace{\left[\begin{array}{ccc}
-\mathrm{i} \omega \mathbf{I} & \mathbf{M}_{\mathbf{0}}^{\prime} & \\
-\mathbf{i} \omega \mathbf{I} & \mathbf{D} \\
\mathbf{D} &
\end{array}\right]}_{\mathbf{A}_{\mathbf{i n c}}}+\alpha \underbrace{\left[\begin{array}{lll}
\mathrm{iM}_{\mathbf{0}} & & \mathrm{iI} \\
& \mathrm{iM}_{\mathbf{0}} & \\
\mathrm{iI} & &
\end{array}\right]}_{\mathbf{B}_{\text {inc }}} \cdot\left[\begin{array}{c}
\mathbf{U} \\
\mathbf{V} \\
\mathbf{P}
\end{array}\right]=0
$$

In the viscous incompressible case, the system given by Eqs. (22-24) becomes quadratic in $\alpha$. To get a system that depends only on the first power of $\alpha$, the two following auxiliary variables are introduced[37]:

$$
\hat{\phi}_{u}=\alpha \hat{u} \quad \text { and } \quad \hat{\phi}_{v}=\alpha \hat{v}
$$

Using the same Chebyshev collocation method as before, the system can then be put in the form of an eigenvalue problem of the type:

$$
\left(\mathbf{A}_{\mathbf{v}}+\alpha \mathbf{B}_{\mathbf{v}}\right)\left[\mathbf{U} \mathbf{V} \mathbf{P} \boldsymbol{\Phi}_{\mathbf{u}} \boldsymbol{\Phi}_{\mathbf{v}}\right]^{t}=0
$$

where $\boldsymbol{\Phi}_{\mathbf{u}}$ and $\boldsymbol{\Phi}_{\mathbf{v}}$ are the vectors containing the $\hat{\phi}_{u}\left(y_{j}\right)$ and the $\hat{\phi}_{v}\left(y_{j}\right)$ at the collocation points. The matrix $\mathbf{A}_{\mathbf{v}}$ and $\mathbf{B}_{\mathbf{v}}$ are now square matrices of size 
$5 N+5$ and are given by:

$$
\mathbf{A}_{\mathbf{v}}=\left[\begin{array}{r|r|r|r|r}
-\mathrm{i} \omega \mathbf{I}-\frac{1}{\mathrm{Re}}\left(\mathbf{N D}^{2}+\mathbf{N}^{\prime} \mathbf{D}\right) & \mathbf{M}_{\mathbf{0}}^{\prime} & & & \\
\hline & -\mathrm{i} \omega \mathbf{I}-\frac{1}{\mathrm{Re}}\left(\mathbf{N D}^{2}+2 \mathbf{N}^{\prime} \mathbf{D}\right) & \mathbf{D} & & \\
\hline \mathbf{D} & & & & \\
\hline & & & \mathbf{I}
\end{array}\right]
$$

$$
\mathbf{B}_{\mathbf{v}}=\left[\begin{array}{c|c|c|c|c}
\mathrm{i} \mathbf{M}_{\mathbf{0}} & -\frac{1}{\mathrm{Re}} \mathrm{i} \mathbf{N}^{\prime} & \mathrm{iI} & \frac{1}{\mathrm{Re}} \mathbf{N} & \\
\hline & \mathrm{i} \mathbf{M}_{\mathbf{0}} & & & \frac{1}{\mathrm{Re}} \mathbf{N} \\
\hline \mathrm{i} \mathbf{I} & & & & \\
\hline-\mathbf{I} & & & & \\
\hline & -\mathbf{I} & & &
\end{array}\right]
$$

The matrix $\mathbf{N}$ and $\mathbf{N}^{\prime}$ are diagonal matrices of size $N+1$ that contain respectively on their diagonal the values of the viscosity $\nu_{T}^{+}\left(y_{j}\right)$ and of the first derivative of the viscosity $\mathrm{d} \nu_{T}^{+} / \mathrm{d} y\left(y_{j}\right)$ at the collocation points. As for the inviscid compressible case, boundary conditions are enforced by replacing the suitable entries of the matrices.

\subsubsection{Shooting methods}

The Chebyshev solution method is a global method that allows obtaining the full spectrum by solving a matrix system in one go. It is very useful but may sometimes be unpractical when the interest lies on a particular eigenvalue and on the tracking of this eigenvalue during the variation of a given parameter. In the later case it is convenient to obtain $\alpha(\omega)$ by a shooting 
method. The shooting method has been used in the compressible and incompressible inviscid cases only, using the Pridmore-Brown equation, Eq. (14), and the Rayleigh equation, Eq. (18), together with boundary conditions (2628) expressed in terms of pressure only. For a fixed value of $\omega$ the shooting method determines the branch $\alpha(\omega)$ in the vicinity of a guessed value of $\alpha$ (which is provided by the Chebyshev method). This method will complement the Chebyshev method occasionally.

\subsubsection{Stability analysis}

The generalized eigenvalue problems (30), (31) and (32) have been derived above. These are of the form:

$$
\mathbf{A}_{\boldsymbol{\omega}} \mathbf{f}=\alpha \mathbf{B}_{\boldsymbol{\omega}} \mathbf{f}
$$

where $\mathbf{A}_{\boldsymbol{\omega}}$ and $\mathbf{B}_{\boldsymbol{\omega}}$ are matrices whose dependence on $\omega$ has been stressed. Solving these problems using standard libraries (in the present case, the function eig of Matlab is used) provides both the eigenvalues and the eigenfunctions. That is, fixing $\omega$, it is possible to obtain the branches $\alpha^{(n)}(\omega)$ satisfying the dispersion relation. Knowing these, it is possible to apply the classical methods of stability such as the Briggs-Bers criterion to classify the modes as stable, convectively unstable, or absolutely unstable [17, 18, 38]. The reader is referred to these references for a detailed discussion of the method; only the essential material is now outlined. First, let us define in the $\omega$-plane a contour $L_{\omega}=\left\{\omega ; \omega_{i}=\right.$ cst $\left.=\omega_{L, i}\right\}$. Such a contour is a line parallel to the real axis. For a well-posed problem and a $L_{\omega}$ contour having a

sufficiently large and positive value of $\omega_{L, i}$, the branches $\alpha^{(n)}(\omega)$ for $\omega \in L_{\omega}$ are well separated: some are lying entirely in the half-plane $\alpha_{i}>0$, the others 
are lying entirely in the half-plane $\alpha_{i}<0$. The method consists in tracking the behaviour of the branches $\alpha^{(n)}(\omega)$ when decreasing the value of $\omega_{L, i}$ from its large initial value towards 0 . There are two possibilities:

- (C1) If in the process of decreasing $\omega_{L, i}$, and before $\omega_{L, i}$ has reached 0 , two opposite spatial branches merge (or pinch) there is an absolute instability consisting of an exponential growth in time in the whole space. By opposite spatial branches, it is meant that one branch is lying entirely in the half-space $\alpha_{i}>0$ while the other is lying entirely in the half-space $\alpha_{i}<0$ for the initial value $\omega_{L, i}$.

- (C2) If such a pinch does not occur there is no absolute instability and the system is at most convectively unstable. It is valid to perform a spatial analysis whereby $\omega$ is real ( $\omega$ then belongs to the $L_{\omega}$ contour having $\omega_{L, i}=0$ ) and the wavenumber is complex. One still needs to identify if there are convectively unstable modes. Any mode having $\alpha_{i} \neq 0$ is a candidate and stability is ultimately determined by the direction of propagation of that mode. To obtain this direction, one has to vary the imaginary part of $\omega$ from 0 to a large value and track by continuity the corresponding branch $\alpha(\omega)$. If, for a very large value of $\omega_{i}$, the mode $\alpha(\omega)$ ends up in the half-plane $\alpha_{i}>0$, this mode is propagating toward $x>0$ (it contributes to the impulse response only in the region $x>0$ ). If it ends up in the half-plane $\alpha_{i}<0$ it is propagating in towards $x<0$. For example suppose a mode has $\alpha_{i}<0$ for real $\omega$ and ends up with $\alpha_{i}>0$ after increasing the imaginary part of $\omega$ to a large value: then this mode would be a convective instability growing towards $x>0$. Both criteria (C1) and (C2) will be used below to determine the kind of stability predicted by the different models. 


\subsubsection{Validation of the numerical solver}

Since the focus will be on unstable modes, it is proposed to validate the method against the solution Rienstra called an unstable hydrodynamic mode[11], which is one of the different surfaces modes for which he obtained an asymptotic analytical expression. In the limit $\omega \rightarrow \infty$, surface waves are solution to the following equation [11]:

$$
\left(1-M_{0} \sigma\right)^{4}=\frac{\left(1-M_{0}^{2}\right)^{3}}{Y^{2}}\left(1-\sigma^{2}\right)
$$

where $\sigma$ is the reduced wave-number defined by: $\alpha=\left(\sigma-M_{0}\right) \omega /\left(1-M_{0}\right)^{2}$. One has to keep only solutions that verifies $\Im(\gamma)>0$, where $\gamma$ is the reduced transverse wavenumber defined by $\gamma=Y\left(1-M_{0} \sigma\right)^{2} /\left(1-M_{0}^{2}\right)^{3 / 2}$, and $\Im$ designates the imaginary part. Initially, results were given by Rienstra in cylindrical coordinates (and for the opposite time convention) but it can be checked that they remain valid in 2D cartesian coordinates. Note also that these results are for a plug flow and the Myers' boundary condition. This is not a problem since we are not interested here in the validity of the boundary condition but only in the validation of the numerical solver.

In Fig. 5, the different modes are given for the following conditions: $M_{0}=0.5$, $\omega=2, Y=2.5$-2.5i. $N=100$ modes are used. Two modes are surface modes as calculated by (36), the one labelled "HI" represents the hydrodynamic instability (according to Rienstra). The precise value obtained for the corresponding wavenumber is given in table 1: the value obtained by the present Chebyshev integration using $N+1=101$ collocation points is in perfect agreement with the asymptotic analysis (which appears to be valid for a value of $\omega$ as low as 2). The agreement is equally good with the shooting method. With a more complicated velocity profile such as the Cess profile that will be 


\begin{tabular}{|l|c|}
\hline \multicolumn{1}{|c|}{ Method } & $\alpha$ \\
\hline $\begin{array}{l}\text { Asymptotic analysis } \\
\text { (Eq. (36)) }\end{array}$ & $7.62064475-2.04970330 \mathrm{i}$ \\
\hline $\begin{array}{l}\text { Numerical integration } \\
\text { Chebyshev using Eq. (30), N=100 }\end{array}$ & $7.62064475-2.04970330 \mathrm{i}$ \\
\hline $\begin{array}{l}\text { Numerical integration } \\
\text { Shooting method using Eq. (14) }\end{array}$ & $7.62064475-2.04970330 \mathrm{i}$ \\
\hline
\end{tabular}

Table 1: Wave-number for the HI mode, as calculated using different methods.

used below, the Chebyshev method requires more points, typically $N=200$.



Figure 5: Spectrum for: $M_{0}=0.5, \omega=2, Y=2.5-2.5 \mathrm{i}$. The plug flow and Myers boundary condition are used together. Symbols: + present numerical method with $N=100$ Chebyshev modes; $o$ asymptotic approximation for surface waves as given by roots of Eq. (36). The numerical value for the Hydrodynamic Instability (HI) mode is given in table 1. 


\section{Results for the inviscid models}

\subsection{Spatial analysis}

Many studies of modes in ducts have involved a spatial stability analysis $[11,12,22]$ whereby the angular frequency is real while the wavenumber is complex. This approach corresponds to point (C2) in section 3.5.3; it is a practical one in the sense that it corresponds to the physical situation whereby one excites the flow at some real frequency. The spatial analysis provides the spatial modes at this frequency. This approach is valid as long as there is no absolute instability, which we are now going to suppose for a moment.

The spatial spectrum is calculated by solving the compressible inviscid problem, Eq. (30). The conditions are identical to the experiment: the flow is approximated by the Cess profile as in Fig. 2, and the angular frequency is the real frequency $\omega=\omega_{a}=0.196$ of the incident wave. The wave number spectrum is complex; it is shown in Fig. 6(a).

Since a convective instability was observed in the experiments, it is appealing to attempt to identify such an instability by applying the criterion (C2) given in section 3.5.3. The imaginary part of the angular frequency is thus increased from 0 to a very large value. Here $\omega$ is varied between $\omega_{a}$ and $\omega_{a}+0.4 \mathrm{i}$; the later value is not extremely large but is sufficient to observe the main move of the spectrum (a further increase does not change the sign of $\alpha_{i}$ ). The $\alpha$-spectra thus obtained are shown in Fig. 6(b). It is seen that only one branch $\alpha$ crosses the $\alpha_{r}$-axis. This branch starts from the value $\alpha \sim 0.79-1.1 \mathrm{i}$ when $\omega=\omega_{a}$ and is indicated by an open square symbol 

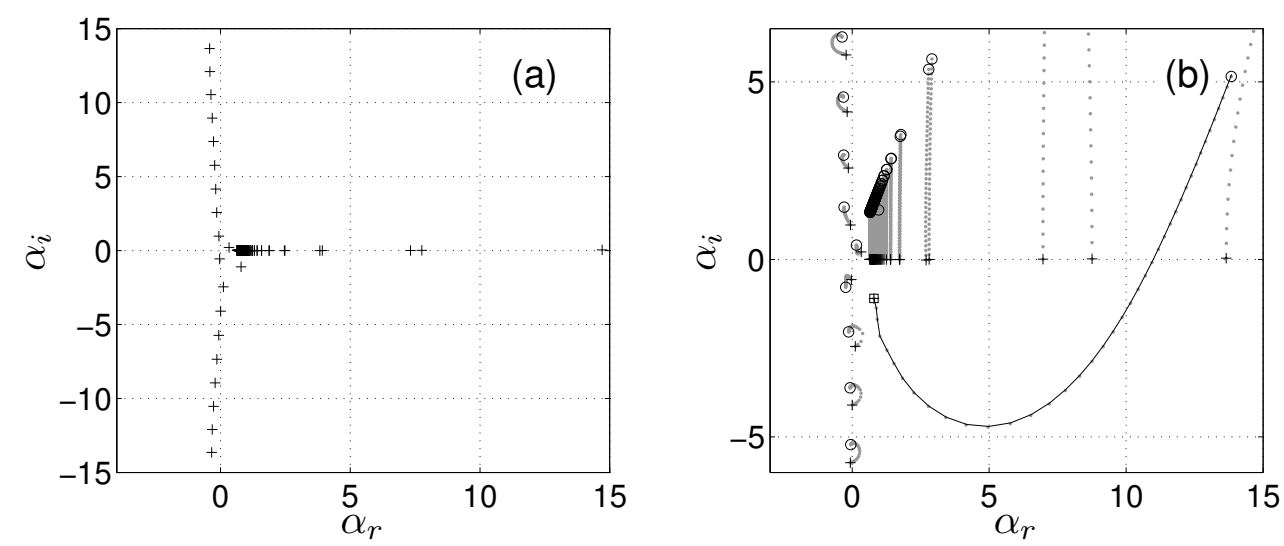

Figure 6: (a) Spectrum calculated by solving (30) for $\omega=\omega_{a}=0.196$. (b) Trajectories of the modes when the imaginary part of $\omega$ is increased from 0 to $0.41 \mathrm{i}$, the real part been kept constant at $\omega_{r}=\omega_{a}$. The symbols + indicate the starting points for $\omega_{i}=0$ (the same as in (a)); the symbols $o$ indicates the points for $\omega_{i}=0.41$. The gray dots are for the trajectory in between. The symbol $\square$ indicates the potentially unstable mode. The line — obtained with the shooting method is a further verification of the trajectory of the potentially unstable mode.

on Fig. 6(b). Hence, if the spatial stability analysis were valid this mode would be a convective instability growing towards $\mathrm{x}>0$ with a growth rate $-\alpha_{i} \sim 1.1$. This reasoning was done in reference [22] assuming that a spatial analysis was valid, that is, assuming that there is no absolute instability. Such a kind of reasoning was also done in [11] with the ill-posed Myers boundary condition. Unfortunately, it is shown below that in our specific case there is an absolute instability, meaning that the spatial analysis is indeed not valid.

\subsection{Spatio-temporal analysis}

In the previous section the criterion (C2) was applied directly without checking condition (C1) (see section 3.5.3). A safer way to investigate sta- 
bility is to start with $L_{\omega}$ contours having a high value of $\omega_{L, i}$, as indicated in condition (C1). Then the value of $\omega_{L, i}$ is decreased and the branches $\alpha$ are monitored. The result is shown in Fig. 7 where only the $\alpha$-branch that is suspected to be an instability has been tracked. This figure features a typical branch swap as $\omega_{L, i}$ is decreased from 0.11 to 0.10 , indicating the presence of a saddle point of the mapping $\alpha(\omega)$. Moreover the branches that swap are opposite in the sense given in $C 1$. This reveals the presence of a pinch point for a positive value of $\omega_{L, i} \sim 0.107$. Hence, there is indeed absolute instability, and the spatial analysis of the previous section was not valid. Note that the sole trajectory, when the imaginary part of $\omega$ is increased, of

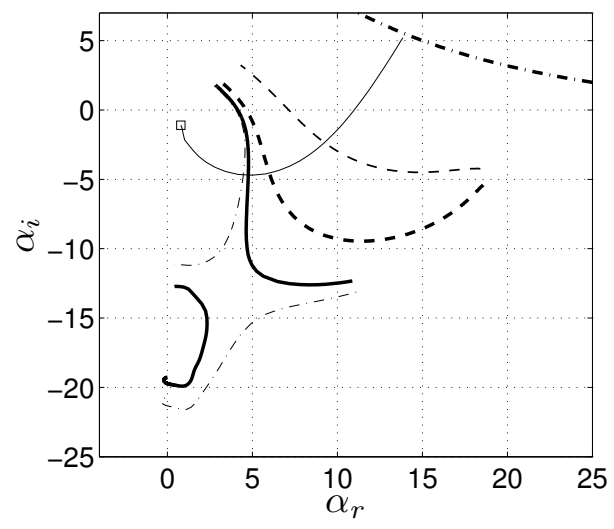

Figure 7: Trajectories of one selected branch $\alpha(\omega)$ for $\omega$ belonging to $L_{\omega}$ contours with: - $\omega_{L, i}=0.10 ;-\omega_{L, i}=0.11 ;-$ - - $\omega_{L, i}=0.12 ;-$ - $\omega_{L, i}=0.18 ;-$ - $\omega_{L, i}=0.41$. The curve — is the trajectory of the branch for $\omega_{r}=\omega_{a}$ and $\omega_{i}$ increasing from 0 to positive values (this is the same as in Fig. 6(b)).

the branch starting at $\alpha\left(\omega_{a}\right) \sim 0.79-1.1 \mathrm{i}$ when $\omega=\omega_{a}$ (square symbol), does not allow identifying such a branch swap in Fig. 6(b). This trajectory is again represented in Fig. 7, and combining this with the mappings $\alpha\left(L_{\omega}\right)$ 
shows that this is indeed the case.

It was shown in $[39,40]$ using a piecewise linear mean flow profile and an inviscid model that absolute instability is unlikely in many practical aeronautical applications. A similar kind of study with a piecewise linear flow and an incompressible inviscid model was performed in a channel for conditions similar to the present study [44]. It concluded that there was an absolute instability of the flow in our configuration. This conclusion remains valid with the present model despite its using a more realistic velocity profile.

\subsection{Incompressible case}

Below, viscous processes are to be taken into account together with an incompressible model. In the present section, the use of an incompressible model is justified by comparing the spectra provided by compressible and incompressible inviscid models. These are respectively given by the solution of Eq. (30) and Eq. (31). The spectra for both models obtained for $\omega=\omega_{a}$ are provided in Fig. 8(a). It is seen that the general agreement is acceptable. In particular the branch that is involved in instability, marked by an arrow, is well described by an incompressible model. Figure 8(b) compares the branches implied in the pinching. The compressible and incompressible models compare very well. The incompressible model predicts absolute instability, and the branch obtained for the contour $\omega_{L, i}=0.12$ (that is, before pinching) are almost indiscernible. This is important since this branch (also marked by an arrow) is basically responsible for instability. Hence, as far as the unstable mode is concerned, the incompressible model seems to be a satisfying approximation. 



Figure 8: (a) Spectra obtained at $\omega=\omega_{a}=0.196$ : + compressible case Eq. (30) ; • incompressible case Eq. (31). (b) Trajectories of the unstable mode along several $L_{\omega}$ contours: - - - $\omega_{L, i}=0.10$ compressible; - $\omega_{L, i}=0.11$ compressible; - - - $\omega_{L, i}=0.12$ compressible; - $\omega_{L, i}=0.10$ incompressible; $-\omega_{L, i}=0.11$ incompressible; - - - $\omega_{L, i}=0.12$ incompressible.

\section{Results for the viscous incompressible model}

In the previous section, the inviscid compressible or incompressible models applied to our experimental configuration predicts absolute instability. This conclusion does not match the experiments, the later rather displaying spatial instability. In the present section, the effect of viscosity is taken back into account, and this will be seen to change the type of instability, from absolute to convective. The model to be used has been presented in section 3.3.

\subsection{Spatially unstable mode}

The spectrum obtained at $\omega=\omega_{a}$ by solving Eq. (32) is given in Fig. 9(a). Some differences are to be noted compared to the spectra obtained at the same frequency with the inviscid, incompressible or compressible, spectra 
displayed in Fig. 8(a). First in the viscous approximation, there is no continuous branch along the $\alpha_{r}$-axis, since the system is no more singular for $\alpha(\omega) U(y)=\omega$. Second there are additional branches due to viscosity located along $\alpha_{r} \sim 0.8$. The evanescent modes along $\alpha_{r}=0$ are present in all cases. Finally, there is a mode with $\alpha_{\text {num }} \sim 0.94-0.22 \mathrm{i}$ indicated by the square symbol, which as before, belongs to an unstable branch. The branch is un-
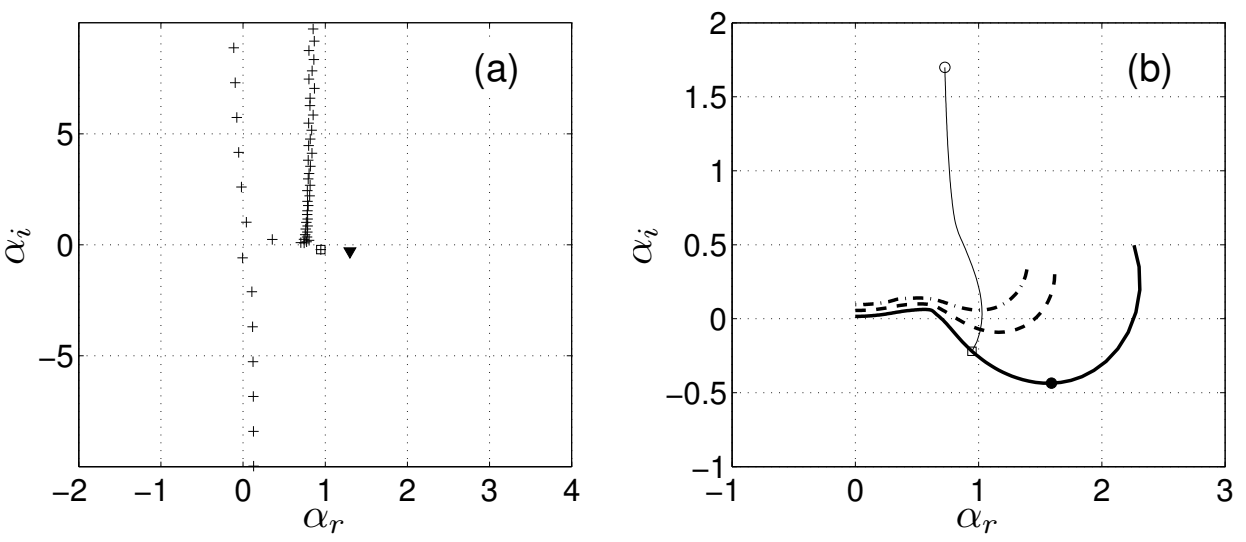

Figure 9: (a) Spectrum obtained at $\omega=\omega_{a}=0.196$ by solving Eq. (32). The symbol $\square$ indicates the convectively unstable mode. The symbol $\mathbf{\nabla}$ indicates the experimental unstable mode. (b) Trajectories of the unstable mode along several $L_{\omega}$ contours: $-\omega_{L, i}=0$ ( $\omega$ real); - - $\omega_{L, i}=0.0009 ;-\cdots \omega_{L, i}=0.0018$. The line — represents the trajectory of the unstable mode when the imaginary part of $\omega$ is increased from 0 ( $\square$ symbol) to $0.41 \mathrm{i}$ ( $o$ symbol), the real part being fixed at $\omega_{r}=\omega_{a}$. The symbol $\bullet$ is for the mode having maximal growth rate for $\omega$ real.

stable because it lies entirely in the upper $\alpha$-plane for large values of $\omega_{i}$, and moves into the lower $\alpha$-plane when $\omega_{i}$ decreases to 0 . This is shown in Fig. 9(b). Contrary to the insviscid case, no pinch has been observed when tracking mappings of $L_{\omega}$ contours in the $\alpha$-plane. Hence, taking into account viscosity does indeed change the stability from absolute instability to convec- 
tive instability. The growth rate $-\alpha_{i}$ for real frequency (contour $\omega_{L, i}=0$ in Fig. 9(b)) is not maximal for $\omega=\omega_{a} \sim 0.196$ ( $\square$ symbol). It has a maximum at $-\alpha_{i} \sim 0.44$ for $\omega_{r} \sim 0.21$ ( symbol). There is a small discrepency here with the experiment since experimentally one would expect the growth rate to be maximal at $\omega=\omega_{a}$. Indeed, in the measurements the acoustic transmission was maximal at this frequency [6], and this should also correspond to a maximal growth rate of the instability. This discrepency is probably due to the idealized modelling as well as to the sensitivity of the growth rate to both flow velocity and frequency (see next subsection).

Here a turbulence model has been used to account for the turbulent flow. Calculations done with a uniform molecular viscosity with a value increased by 30 times gave comparable results. Interestingly, Reynolds and Hussain [31] also found that their spatial modes calculated either with an eddy viscosity model or with a uniform viscosity multiplied by 30 were very similar. Hence, for preliminary studies about the effect of viscosity on incompressible modes in a channel, one could possibly use a molecular viscosity multiplied by 30 .

In conclusion the instability is a spatial one, growing in the $x$-direction with a growth rate $-\alpha_{i}=0.22$. The computed value $\alpha_{\text {num }} \sim 0.94-0.22 \mathrm{i}$ has to be compared to the experimental value $\alpha_{\exp }=1.3-0.3 \mathrm{i}[6]$. This is less than a $30 \%$ error, which is acceptable given the approximations that have been made. 
The numerical eigenfunction corresponding to this unstable mode are also provided by the solution of Eq. (32). They are compared to the eigenfunctions measured experimentally[6] in Fig. 10(a-d). The amplitudes are normalized with respect to the maximum of the transverse velocity and the phase is normalized with respect to the phase of the axial velocity at the channel center. There is a good agreement between the numerical and experimental eigenfunctions, with an error more pronounced on the axial velocity magnitude.

\subsection{Effect of mean flow velocity}

The only parameter that could be changed during the experiments reported in reference [6] was the flow Mach number. While the laser velocity measurements were performed only at $M_{0, \text { avg }}=0.27$, acoustic transmission were measured over a range of Mach number below this value. Indeed, the instability was first uncovered from high acoustic transmission rates by the liner [6, Fig. 5]. Sound transmission by the liner displays a peak for Mach numbers larger than about 0.2. Above this value, the frequency for maximal transmission increases with Mach number. If we suppose that the sound transmission is proportional to the unstable wave amplification rate, this means that: an unstable wave should exist only above some threshold value of the Mach number; and past this value the frequency for maximal amplification should increase. To test these statements, the effect of velocity is now investigated. Notice that the model is incompressible, but the mean sectionaveraged velocity is normalized by the speed of sound in the compressible fluid and is thus presented as a section-averaged Mach number, $M_{0, \text { avg }}$. In the mean velocity range investigated no pinching point has been observed and the instability is spatial whenever it exists, which legitimates a spatial 

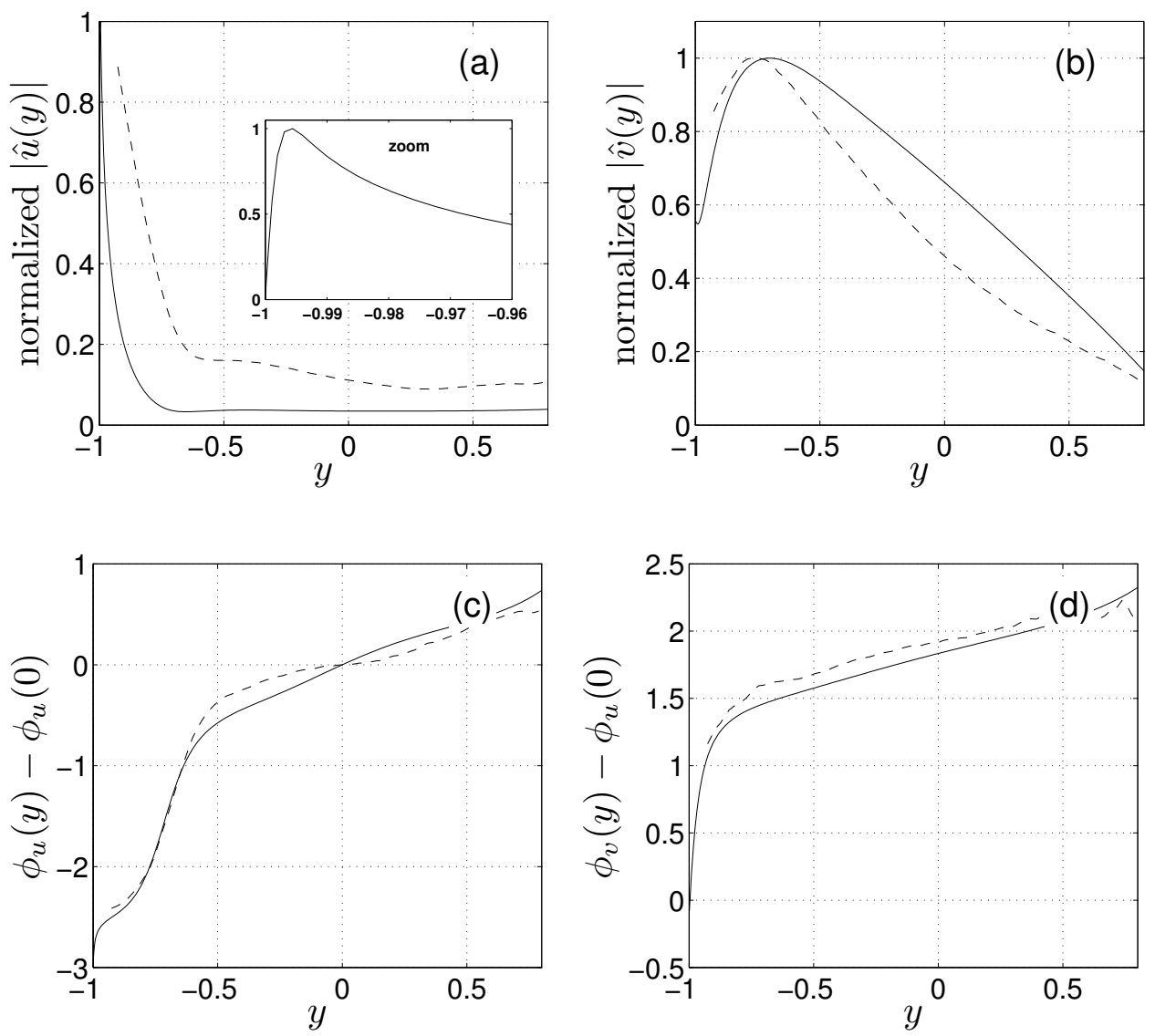

Figure 10: Comparison of numerical ( - ) and experimental (-- ) eigenfunctions for the unstable mode at $\omega_{r}=\omega_{a}$. (a) Amplitude of the axial component; (b) Phase of the axial component; (c) Amplitude of the transverse component; (d) Phase of the transverse component.

stability analysis.

First, the unstable mode wavenumber is tracked when the Mach number is increased, keeping frequency constant at the baseline experimental value: $\omega=\omega_{a}=0.196$. The trajectory of the mode in the $\alpha$-plane is shown 
in Fig. 11(a). For low values of the Mach number the wavenumber lies in the upper $\alpha$-plane and it moves into the lower $\alpha$-plane for a sufficiently high value of the Mach number. It can be shown that the trajectory lies entirely in the upper $\alpha$-plane if the frequency is given a large positive imaginary part value, which means (criterion (C2)) that when $\alpha_{i}<0$ the mode is a spatial instability. The spatial growth rate is shown in Fig. 11(b) as a function of Mach number. At the chosen frequency, the mode is thus unstable for values of the Mach number larger than about 0.17.
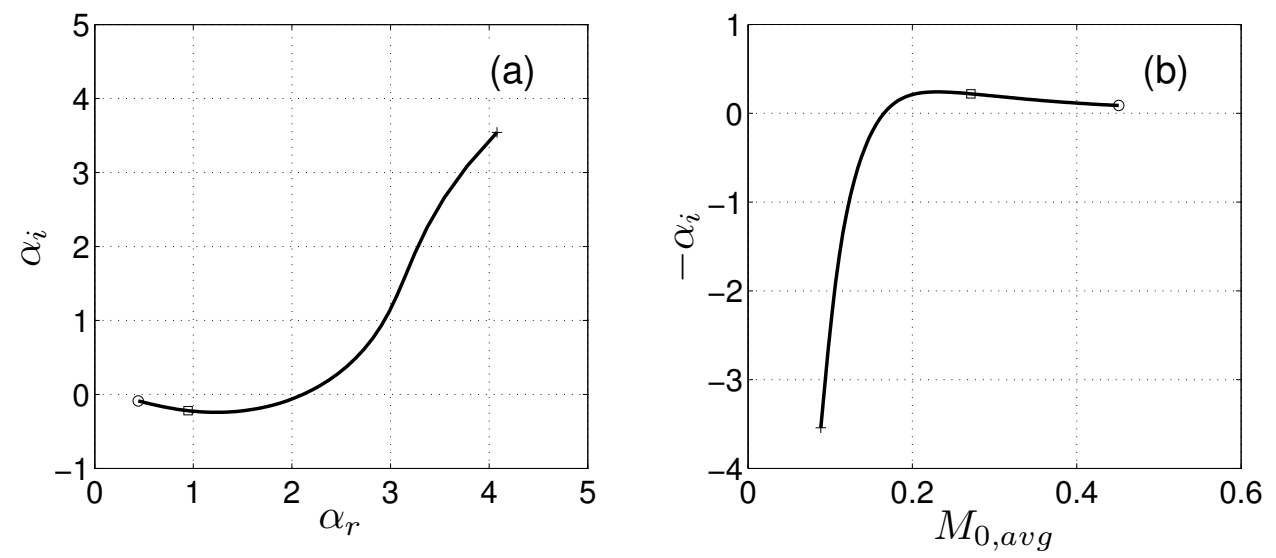

Figure 11: (a) Trajectory of the unstable mode and (b) Growth rate as a function of the section-averaged Mach number, obtained at $\omega=\omega_{a}=0.196$ when varying the sectionaveraged Mach number from $M_{0, \text { avg }} \sim 0.09$ ( + symbol) to $\sim 0.45$ ( $o$ symbol). The symbol $\square$ indicates the convectively unstable mode at the baseline value $M_{0, \text { avg }}=0.27$.

A wider picture is obtained by varying the (real) frequency for several values of the Mach number. The growth rate obtained for the mode is shown in Fig. 12. For the lowest Mach number, $M_{0, \mathrm{avg}}=0.16$, the growth rate is negative for all frequencies, and there is no instability. For all Mach number 
above this threshold of about $M_{0, \text { avg }} \sim 0.16$, there is spatial instability over some frequency range. The frequency range sustaining instability is wider for larger Mach numbers. One observes also that the maximum of the growth rate is shifting towards higher values of $\omega_{r}$ when the Mach number is increased. Hence, the model allows to confirm the experimental observations mentioned above.

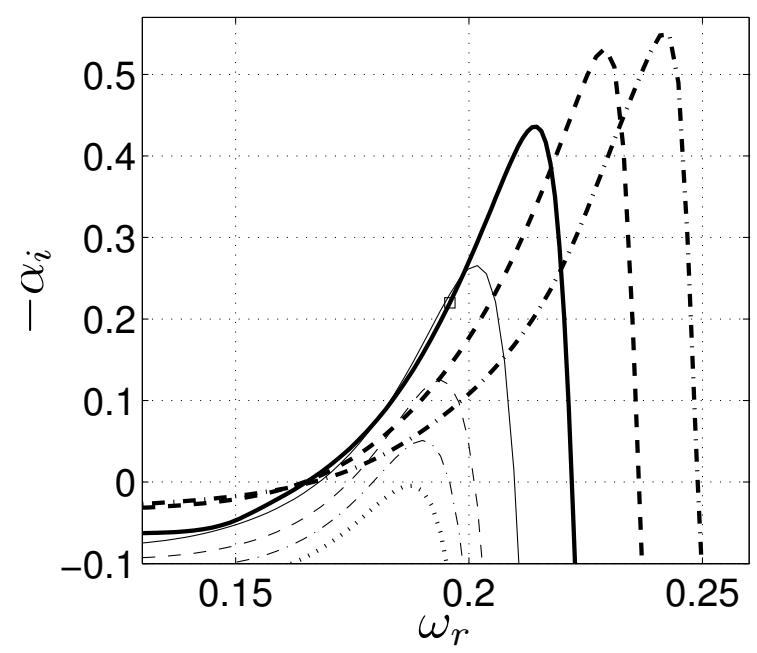

Figure 12: Spatial growth rate for the spatially unstable branch as a function of $\omega_{r}$ for several values of the section-averaged Mach number: $\ldots M_{0, \text { avg }}=0.16 ;-\cdots M_{0, \text { avg }}=0.165 ;$ - - $M_{0, \text { avg }}=0.18 ;-M_{0, \text { avg }}=0.21 ;-M_{0, \text { avg }}=0.27 ;-\ldots M_{0, \text { avg }}=0.36 ; \ldots M_{0, \text { avg }}=0.45$. The symbol $\square$ indicates the convectively unstable mode at the baseline value $M_{0, \text { avg }}=0.27$ and $\omega=\omega_{a}=0.196$.

\section{Conclusion}

A local, two-dimensionnal, linear stability analysis of the flow in a lined channel has been performed. The acoustic liner covers the bottom wall of 
the channel and is represented by an impedance model. The mean flow profile is representative of the flow in a turbulent channel. Several models have been used to compute the modes: an inviscid compressible model, an inviscid incompressible model, and finally a viscous incompressible model. In the later, turbulence is taken into account by an eddy viscosity model. The inviscid models both show an instability that does not depend much on the compressibility, and the instability is of the absolute type. When taken into account, viscosity changes the type of stability, from absolute to convective instability.

The conditions taken for the analysis are similar to that of a previsouly reported experiment: same geometry, same impedance law, same real frequency for spatial analyses, same mean flow Mach number. There are necessary simplifications: in particular, the analytical velocity profile corresponds well to the flow in a channel with rigid walls. An expression for velocity profiles (that is, equivalently, an expression for the eddy viscosity) over lined wall would be desirable. Despite these simplifications, the viscous model identifies a spatial instability whose characteristics does match correctly the experimental ones, in terms of wavenumber (wavelength, and growth rate) and of eigenfunction shapes.

\section{References}

[1] A. W. Guess, Calculation of perforated plate liner parameters from specified acoustic resistance and reactance, Journal of Sound and Vibration 40(1) (1975) 119-137. 
[2] R. E. Motsinger, R. E. Kraft, Design and performance of duct acoustic treatment, Aeroacoustics of Flight Vehicles, Edited by H H Hubbard, Acoustical Society of America, Vol. 2, 165-206, 1995.

[3] E. Meyer, F. Mechel, G. Kurtze, "Experiments on the influence of flow on sound attenuation in absorbing ducts", Journal of the Acoustical Society of America 30 (1958) 165-174.

[4] M. Brandes, D. Ronneberger, Sound Amplification in flow ducts lined with a periodic sequence of resonators, Proceedings of the first AIAA/CEAS aeroacoustic conference, Munich, Germany, AIAA Paper 95-126, 1995.

[5] Aurégan Y., Leroux M., "Experimental evidence of an instability along an impedance wall with flow", Journal of Sound and Vibration, 317 (2008) 432-439.

[6] D. Marx, Y. Aurégan, H. Bailliet, J.-C. Valière, PIV and LDV evidence of hydrodynamic instability over a liner in a duct with flow, Journal of Sound and Vibration 329 (2010) 3798-3812. doi: 10.1016/j.jsv.2010.03.025

[7] X. Li, C. Richter, F. Thiele, Time-domain impedance boundary conditions for surfaces with subsonic mean flows, Journal of the Acoustical Society of America 119(5) (2006) 2665-2676.

[8] M. O. Burak, M. Billson, L.-E. Eriksson, S. Baralon, Validation of a Time and Frequency Domain Grazing Flow Acoustic Liner Model, AIAA Journal 47(8) (2009) 1841-1848. 
[9] B. J. Tester, The propagation and attenuation of sound in lined ducts containing uniform or plug flow, Journal of Sound and Vibration 28(2) (1973) 151-203.

[10] W. Koch, W. Mohring, Eigensolutions for liners in uniform mean flow ducts, AIAA Journal 21(2) (1983) 200-213. doi:10.2514/3.8055

[11] S. W. Rienstra, A classification of duct modes based on surface waves, Wave motion 37 (2003) 119-135. doi:10.1016/S0165-2125(02)00052-5

[12] E. J. Brambley, N. Peake, Classification of aeroacoustically relevant surface modes in cylindrical lined ducts, Wave motion 43 (2006) 301-310. doi: 10.1016/j.wavemoti.2006.01.001

[13] U. Ingard, Influence of fluid motion past a plane boundary on sound reflection, absorption, and transmission, Journal of the Acoustical Society of America 31(7) (1959) 1035-1036.

[14] M. K. Myers, On the acoustic boundary condition in the presence of flow, Journal of Sound and Vibration 71(3) (1980) 429-434. doi: 10.1016/0022-460X(80)90424-1

[15] S. Rienstra, G. Vilenski, Spatial instability of boundary layer along impedance wall, Proceedings of the 14 th AIAA/CEAS aeroacoustic conference, Vancouver, Canada, AIAA Paper 2008-2932, 2008.

[16] E. J. Brambley, Fundamental problems with the model of uniform flow 
over acoustic linings, Journal of Sound and Vibration 322 (2009) 10261037.

doi:10.1016/j.jsv.2008.11.021

[17] R. J. Briggs, Electron-Stream Interaction with Plasmas, MIT Press, Cambridge, 1964, chap 2.

[18] P. Huerre, M. Rossi, Hydrodynamic Instabilities in Open Flows, Hydrodynamics and Nonlinear Instabilities, Edited by C. Godrèche and P. Manneville, Cambridge University Press, 1998, pp. 81-294.

doi: 10.1017/CBO9780511524608.004

[19] P. N. Shankar, Acoustic refraction and attenuation in cylindrical and annular ducts, Journal of Sound and Vibration 22(2) (1972) 233-246.

[20] N. K. Agarwal, M. K. Bull, Acoustic wave propagation in a pipe with fully developed turbulent flow, Journal of Sound and Vibration 132(2) (1989) 275-298.

[21] Kousen, K. A., Eigenmodes of ducted flows with radially-dependent axial and swirl velocity components, NASA CR-1999-208881, 1999.

[22] D. Marx, Y. Aurégan, Comparison of experiments with stability analysis predictions in a lined flow duct, Proceedings of the 16th AIAA/CEAS aeroacoustic conference, Stockholm, Sweden, AIAA Paper 2010-3946, 2010.

[23] G. Boyer, E. Piot, J.-P. Brazier, Theoretical investigation of hydrodynamic surface mode in a lined duct with sheared flow and comparison 
with experiments, Journal of Sound and Vibration 330 (2011) 1793-1809. doi: 10.1016/j.jsv.2010.10.035

[24] P O A L Davies, M Bhattacharya, J L Bento Coehlo, Measurement of plane wave acoustic fields in flow ducts (1980), Journal of Sound and Vibration 72(4) 539-542.

[25] M C A M Peters, A Hirschberg, A J Reijnen, A P J Wijnands, Damping and reflection reflection coefficient measurements for an open pipe at low Mach and low Helmholtz numbers Journal of Fluid Mechanics 265 (1993) 499-534.

[26] M S Howe, The damping of sound by wall turbulent shear layers Journal of the Acoustical Society of America 98(3) (1995) 1723-1730.

[27] E Dokumaci, A note on transmission of sound in a wide pipe with mean flow and viscothermal attenuation, Journal of Sound and Vibration 208(4) (1997) 653-655.

[28] S Allam, M Abom, Investigation of damping and radiation using full plane wave decomposition in ducts, Journal of Sound and Vibration 292 (2006) 519-534.

[29] Y Aurégan, R Starobinski, V Pagneux, Influence of grazing flow and dissipation effects on the acoustic boundary conditions at a lined wall, Journal of the Acoustical Society of America 109(1) (2001) 59-64.

[30] W. C. Reynolds, W. G. Tiederman, Stability of turbulent channel flow, with application to Malkus's theory, Journal of Fluid Mechanics 27(2) (1967) 253-272. 
[31] W. C. Reynolds, A.K. M. F. Hussain, The mechanics of an organized wave in turbulent shear flow. Part 3: Theoretical models and comparisons with experiments, Journal of Fluid Mechanics 54(2) (1972) 263288.

[32] G. Pujals, M. Garcia-Villalbva, C. Cossu, S. Depardon, A note on optimal transient growth in turbulent channel flows, Physics of fluids 21 (2009) 015109.

[33] D. Marx, Y. Aurégan, H. Bailliet, J.-C. Valière, Evidence of Hydrodynamic instability over a liner in a duct with flow, 15th AIAA/CEAS aeroacoustics conference, Miami, Florida, 11-13 may 2009, AIAA PAPER 2009-3170.

[34] S W Rienstra, Impedance models in time domain including the extended Helmholtz resonator model (2006), Proceedings of the 12th AIAA/CEAS aeroacoustic conference, Cambridge, USA, AiAA Paper 2006-2686.

[35] Trefethen, L. N., Spectral methods in Matlab, SIAM, 2001.

[36] Canuto, C., Hussaini, M. Y., Quarteroni, A., Zang, T. A., Spectral Methods in Fluid Dynamics, Springer, 1987.

[37] Bridges, T. J., Morris, P. J., Differential eigenvalue problems in which the parameter appears nonlinearly, Journal of Computational Physics 55 (1984) 437-460.

[38] P. Huerre, P. Monkewitz, Absolute and convective instabilities in free shear layers, Journal of Fluid Mechanics 159 (1985) 151-168. doi: $10.1017 / \mathrm{S} 0022112085003147$ 
[39] S. W. Rienstra, M. Darau, Mean flow boundary layer effects of hydrodynamic instability of impedance wall, IUTAM Symposium on Computational Aero-Acoustics for aircraft noise prediction, Procedia engineering, Vol. 6, 2010, pp. 124-132.

doi: 10.1016/j.proeng.2010.09.014

[40] S. W. Rienstra, M. Darau, Boundary-layer thickness effects on the hydrodynamic instability along an impedance wall, Journal of Fluid Mechanics 671 (2011) 559-573. doi:10.1017/S0022112010006051

[41] E. Brambley, Acoustic implications of a thin viscous boundary layer over a compliant surface or permeable liner, Journal of Fluid Mechanics 678 (2011) 348-378.

[42] E. Brambley, Well-Posed Boundary Condition for Acoustic Liners in Straight Ducts with Flow, AIAA Journal 49(6) (2011) 1272-1282.

[43] Y. Renou, Y. Aurégan, Failure of the Ingard Myers boundary condition for a lined duct: An experimental investigation, Journal of the Acoustical Society of America, 130(1) (2011) 52-60.

[44] D. Marx, A piecewise linear mean flow model for studying stability in a lined channel, Journal of Sound and Vibration 331 (2012) 3809-3823. 